\title{
Frequency-dependent changes in the amplitude of low-frequency fluctuations in patients with Wilson's disease: a resting-state fMRI study
}

\author{
Xiaopeng $\mathrm{Hu}^{1}$ • Siyi Chen ${ }^{2,3}$ - Chang-Bing Huang ${ }^{2} \cdot$ Yinfeng Qian $^{1}$ - Yongqiang Yu ${ }^{1}$
}

Received: 30 July 2016 / Accepted: 26 December 2016/Published online: 24 January 2017

(C) The Author(s) 2017. This article is published with open access at Springerlink.com

\begin{abstract}
To investigate the frequency-dependent changes in the amplitude of low-frequency fluctuations (ALFF) in patients with Wilson's disease (WD). Resting-state function magnetic resonance imaging (R-fMRI) were employed to measure the amplitude of ALFF in 28 patients with WD and 27 matched normal controls. Slow-5 $(0.01-0.027 \mathrm{~Hz})$ and slow-4 $(0.027-0.073 \mathrm{~Hz})$ frequency bands were analyzed. Apart from the observation of atrophy in the cerebellum, basal ganglia, occipital gyrus, frontal gyrus, precentral gyrus, and paracentral lobule, we also found widespread differences in ALFF of the two bands in the medial frontal gyrus, inferior temporal gyrus, insula, basal ganglia, hippocampus/ parahippocampal gyrus, and thalamus bilaterally. Compared to normal controls, WD patients had increased ALFF in the posterior lobe of the cerebellum, inferior temporal gyrus, brain stem, basal ganglia, and decreased ALFF in the anterior lobe of the cerebellum and medial frontal gyrus. Specifically, we observed that the ALFF abnormalities in the cerebellum and middle frontal gyrus were greater in the slow- 5 than in the slow-4 band. Correlation analysis showed consistently positive correlations between urinary copper excretion $(\mathrm{Cu})$,
\end{abstract}

Xiaopeng Hu and Siyi Chen contributed equally to the manuscript.

Yinfeng Qian

894206876@qq.com

Yongqiang $\mathrm{Yu}$

Cjr.yuyongqiang@vip.163.com

1 Department of Radiology, First Affiliated Hospital of Anhui Medical University, Hefei 230022, China

2 Key Laboratory of Behavioral Science, Institute of Psychology, Chinese Academy of Sciences, Beijing 100101, China

3 Department Psychologie, Ludwig-Maximilians-Universität, 80802 Munich, Germany serum ceruloplasmin (CP) and ALFFs in the cerebellum. Our study suggests the accumulation of copper profoundly impaired intrinsic brain activity and the impairments seem to be frequency-dependent. These results provide further insights into the understanding of the pathophysiology of WD.

Keywords Wilson's disease $\cdot$ Resting-state functional magnetic resonance imaging $\cdot$ Amplitude of low-frequency fluctuations

\section{Background}

Wilson's disease (WD) or hepatolenticular degeneration, is first defined by Alexander Kinnier Wilson in 1912 (Wilson 1912). The physical burden of the disease falls largely in the liver and brain (Reilly et al. 1993). Accumulation of cooper in the liver can lead to elevated serum aminotransferases, chronic hepatitis, and cirrhosis (Ala et al. 2007). In the brain, most copper is deposited in the basal ganglia, particularly in the lenticular nucleus, which areas normally participate in action selection, movement control, procedural and reward learning, and emotion regulation (Yamada et al. 2004; Turner et al. 2003; Packard and Knowlton 2002; Radua et al. 2010). About half the people with Wilson's disease will develop neurological or psychiatric symptoms, such as mild cognitive deterioration in the early stage (de Bie et al. 2007), and dysarthria, abnormal gait, risus sardonicus, dystonia, rigidity, bradykinesia in the late stages (Machado et al. 2006), indicating potential brain changes in WD patients. Previous studies have revealed generalized brain atrophy, lesions in the brainstem, cerebellum, basal ganglia, subcortical white matter, midbrain, and pons of WD patients with conventional magnetic resonance imaging (MRI) (Page et al. 2004; Sinha et al. 2006; 
Magalhaes et al. 1994; Starosta-Rubinstein et al. 1987), diffusion or focal hypoperfusion in superior frontal, prefrontal, parietal, occipital cortices, in temporal gyri, caudatus and putamen with single photon emission computed tomography (SPECT) studies (Piga et al. 2008), and decreased cerebellar, striatal and cortical and thalamic regional cerebral metabolic rate of glucose consumption (rCMRGlc) with positron emission tomography (PET) (Kuwert et al. 1992; Hawkins et al. 1987). In the current study, we collected both structural and resting-state imaging data in WD patients and normal controls, aiming to provide additional information other than structural changes about how WD will affect brain.

In the last two decades, reliable and easy-to-perform resting-state functional magnetic resonance imaging (RfMRI) has attracted more and more attention in the diagnosis of brain activities since Biswal and his colleagues (Biswal et al. 1995) first reported that spontaneous low-frequency oscillations (LFO; $0.01-0.08 \mathrm{~Hz}$ ) of blood oxygen level-dependent (BOLD) signal were physiologically informative. Amplitude of low-frequency fluctuations (ALFF), characterizing the local brain features of RfMRI data, is one of the most frequently used approaches that quantify the regional neural synchronous activity by assessing the amplitude of power spectrum of LFO (Zang et al. 2007). The ALFF analysis has been used to detect brain abnormalities in a spectrum of diseases, such as the attentiondeficient hyperactivity disorder (Zang et al. 2007), Alzheimer's disease (He et al. 2007), and posttraumatic stress disorder (Yin et al. 2011). While resting-state PET/SPECT directly measures the metabolism of local brain areas, the ALFF measures the deviation of the BOLD signal and may provide extra information about how disease affects brain.

In the present study, we utilized ALFF of R-fMRI to examine WD-related abnormal changes in LFO amplitudes. We computed ALFF at the slow-5 (0.01-0.027 Hz) and slow-4 bands $(0.027-0.073 \mathrm{~Hz})$ to evaluate if the WDrelated deficits were frequency-specific. Stronger ALFF at slow-5 tended to be present in the cortical structures, and stronger slow-4 tended to be present in the subcortical structures (Zou et al. 2010). Moreover, several diseases have been shown to be band specific (Hoptman et al. 2010; Wang et al. 2014). Since other frequency bands, e.g. slow-6 (0-0.01 Hz), slow-3 $(0.073-0.198 \mathrm{~Hz})$, and slow-2 $(0.198-0.25 \mathrm{~Hz})$, may mingle with very low frequency drift, the white matter (WM) signals, and highfrequency physiological noises, they were not analyzed (Biswal et al. 1995; Zou et al. 2010). We also investigated the associations between ALFF and biochemical measures, e.g. 24-h urinary copper excretion $(\mathrm{Cu})$ and serum ceruloplasmin (CP), aiming to confirm the relationship between ALFF measures and clinical diagnosis.

\section{Method}

\section{Subjects}

Twenty-eight neurological WD patients (18 males), with $21.43 \pm 3.56$ years (mean \pm s.d.), were recruited from the Institute of Neurology at Anhui University of Chinese Medicine, Hefei, China. The mean duration of illness was 6.67 ( \pm 1.46 , s.d.) years when they participated the study. Neurological WD patients were hospitalized and were intravenously administered sodium dimercaptopropanesulfonate (DMPS) at a dose of $15-20 \mathrm{mg} / \mathrm{kg}$ throughout the course of the treatment to promote the urinary excretion of copper. Each treatment course consisted of DMPS therapy for 6 consecutive days, followed by a withdrawal period of 2 days to promote tolerance. Every patient received repeated DMPS therapy for 8 to 10 courses every year. All patients demonstrated neurological improvements and decreased urinary copper excretion after 6 courses but experienced worsening of symptoms after 6 months to 1 year post DMPS therapy. All neurological WD patients who participated in this study didn't receive any treatments during the past 6 months before this study. They began the treatment after the procedure of diagnosis and scanning. The diagnosis was made based on clinical symptoms (e.g., presentation of extrapyramidal symptoms and signs, with dysarthria and tremor, and remarkable impairment of liver function), the presence of the Kayser-Fleischer Ring (the KF ring), and abnormal copper metabolism (decreased levels of serum ceruloplasmin: $\mathrm{CP}<20 \mathrm{mg} / \mathrm{dL}$; increased 24-h urinary excretion of copper: 24-h urinary $\mathrm{Cu}>1.6 \mu \mathrm{mol} /$ day) (European Association For The Study Of The Liver 2012). The average 24-h urinary $\mathrm{Cu}$ was $2.42 \pm 1.48 \mu \mathrm{mol} /$ day (mean \pm s.d.) and the average CP was $6.15 \pm 2.51 \mathrm{mg} / \mathrm{dL}$ (mean \pm s.d.) based on the group of neurological WD patients. Twenty-seven normal controls (NC; 15 males), aged $23.41 \pm 2.65$ years (mean \pm s.d.), were recruited from the local community. Subjects (patients and normal controls) with history of head injury, neurological disorders, or concomitant medical disorders were excluded. All subjects, with written informed consents, were scanned within $24 \mathrm{~h}$ of initial contact. This study was approved by the Medical Research Ethics Committee of Anhui Medical University. The characteristics of all subjects were summarized in Table 1.

\section{MRI data acquisition}

All subjects were scanned on a GE HDxt (General Electric Medical Systems, Milwaukee, WI, USA) 3.0 T magnetic resonance scanner at the first-affiliated hospital of Anhui Medical University. Resting-state fMRI was obtained using an 480-s EPI (echo planar imaging) pulse sequence: TR (repetition time $)=2000 \mathrm{~ms}, \mathrm{TE}$ (echo time $)=30 \mathrm{~ms}$, flip angle $=90^{\circ}$, field of view $=240 \times 240 \mathrm{~mm}^{2}$, voxel size $=3.75 \times 3.75 \times 3.75 \mathrm{~mm}^{3}$, matrix $=64 \times 64$, number of slices $=39$, slice thickness $=3 \mathrm{~mm}$, 
Table 1 Characteristics of the participants

\begin{tabular}{lll}
\hline & WD $(N=28)$ & NC $(N=27)$ \\
\hline Gender (male/female) & $18 / 10$ & $15 / 12$ \\
Age (years) & $16-27(21.43 \pm 3.56)$ & $17-31(23.41 \pm 2.65)$ \\
Education (years) & $8-14(10.19 \pm 1.33)$ & $9-15(11.02 \pm 2.52)$ \\
Handedness & 28 right-handed & 27 right-handed \\
WD duration (years) & $4-9(6.67 \pm 1.46)$ & \\
WD types & neurologic & \\
The KF ring & 28 WD with the KF ring & \\
$24-h$ urinary Cu $(\mu \mathrm{mol} /$ day $)$ & $1-6(2.42 \pm 1.48)(N=19)$ & \\
$\mathrm{CP}(\mathrm{mg} / \mathrm{dL})$ & $3.43-13.02(6.15 \pm 2.51)(N=19)$ & \\
\hline
\end{tabular}

$W D$ Wilson's disease, $N C$ Normal controls, $N$ Number, $K F$ Kayser-Fleischer, $C u$ Copper, $C P N$ Ceruloplasmin gap $=0.8 \mathrm{~mm}$. For structural imaging, a 3-dimensional spoiled gradient-recalled acquisition (3D-SPGR) technique was used: $\mathrm{TR}=7.012 \mathrm{~ms}, \mathrm{TE}=2.876 \mathrm{~ms}, \mathrm{TI}=900$ $\mathrm{ms}$, flip angle $=8^{\circ}$, field of view $=256 \times 256 \mathrm{~mm}^{2}$, voxel size $=0.94 \times 0.94 \times 1.2 \mathrm{~mm}^{3}$, matrix $=256 \times 256$, slice thickness $=1 \mathrm{~mm}$ without gap, 166 slices.

\section{Data preprocessing}

The preprocessing was carried out using Data Processing Assistant for Resting-State fMRI (DPARSF, http://www. restfmri.net/) (Chao-Gan and Yu-Feng 2010), which is based on Statistical Parametric Mapping (SPM8, http://www.fil.ion. ucl.ac.uk/spm/) and Resting-State fMRI Data Analysis Toolkit (REST, http://www.restfmri.net/) (Song et al. 2011). First ten volumes were discarded to allow MRI signal to reach a steady state and the subjects to get used to the scanner environment. The remaining fMRI images were slice-acquisition and head-motion corrected with a least-square approach and a six-parameter spatial transformation. Three WD patients and one normal control were excluded according to the criteria that head motion was restricted to less than $2 \mathrm{~mm}$ of displacement or 2 degrees of rotation in any directions. Six motion parameters, the cerebrospinal fluid (CSF), and the white matter signals were used as nuisance covariates to reduce the effects of head motion and nonneuronal BOLD fluctuations (Biswal et al. 1995; Zou et al. 2010 ). All functional data were normalized to the standard SPM8 Montreal Neurological Institute (MNI) template by applying the transformation parameters obtained from the structural images to those corrected images, and then resampled to $3-\mathrm{mm}^{3}$ voxels. The resultant normalized functional images were spatially smoothed with a Gaussian kernel of 4-mm full width at half maximum (FWHM) and removed of linear trends.

\section{ALFF analysis}

We used REST software to calculate ALFF (Zang et al. 2007; Song et al. 2011). The time series for each voxel was first band- pass filtered $(0.01-0.027 \mathrm{~Hz}$ for slow-5 band, and 0.027 $0.073 \mathrm{~Hz}$ for slow-4). The filtered time series was fast Fourier transform (FFT) transformed to obtain power spectrum in the frequency domain (parameters: taper percent 0 , FFT length shortest). The square root of the power spectrum was computed and averaged across pre-defined frequency intervals at each voxel. This averaged square root was taken as the ALFF at a particular frequency band. To reduce the effects of participants' variability, ALFF of each voxel for each participant was further divided by the global mean ALFF (Zang et al. 2007). The global mean ALFF value was calculated for each participant within a group gray matter (GM) mask obtained by selecting a threshold of 0.2 on the mean GM map of all 51 subjects.

\section{Structural image analysis}

The loss of GM in WD may confound the ALFF analysis. To identify the brain regions with GM loss, we performed a voxel-based morphometry (VBM) analysis for structural images (http://www.fil.ion.ucl.ac.uk/spm). Briefly, individual structural images (3D T1-weighted anatomical images) were co-registered to the mean functional images after motion correction using a linear transformation (Collignon et al. 1995). The transformed structural images were then segmented and spatially normalized into GM, WM, and cerebrospinal fluid in the MNI space by using a unified segmentation algorithm (Ashburner and Friston 2005). Individual GM maps were further modulated to compensate for the effect of spatial normalization using both linear and nonlinear methods. The GM images underwent a spatial smoothing using 4-mm full width at half maximum (FWHM) Gaussian kernel. The resultant images were used to identify the brain regions with GM loss. Moreover, these maps were also taken into the following statistical analysis to examine the effects of GM atrophy on the functional results. In the study, we utilized the mean GM map (threshold $=0.2$ ) to generate a group-based GM mask and used this mask for analyzing ALFF differences between the normal and WD groups. 


\section{Statistical analysis}

A voxel-based two-sample t-test was applied on the smoothed GM intensity maps to determine the results of GM loss in the WD patients. We performed a two-way repeated-measure analysis of variance (ANOVA) on a voxel-by-voxel basis with group (WD patients and normal controls) as a between-subject factor and frequency band (slow-4 and slow-5) as withinsubject factors in which the GM intensity maps were entered as covariates. Monte Carlo simulation was utilized to correct for multiple comparisons by using the Resting-State fMRI Data Analysis Toolkit (version 1.8) (Song et al. 2011). A corrected significance level of $P=0.05$ was obtained by a combined threshold of $P=0.01$ for each voxel and an extent threshold of 66 voxels (cluster size $>1782 \mathrm{~mm}^{3}$ ). The correlation analyses were conducted after GM correction. Spearman's correlation coefficient was used to study the association between LFO amplitudes and biochemical parameters, i.e., $\mathrm{Cu}(\mu \mathrm{mol} / \mathrm{day})$ and $\mathrm{CP}(\mathrm{mg} / \mathrm{dL})$.

\section{Results}

\section{GM volume}

VBM analysis revealed decreased GM volume in the basal ganglia, bilateral cerebellum, right superior and left middle occipital gyrus, right middle frontal gyrus, bilateral medial frontal gyrus, left precentral gyrus, left paracentral lobule, and increased GM volume in bilateral parahippocampal gyrus (Fig. 1, Table 2).
ALFF (slow 5: 0.01-0.027 Hz, slow 4: 0.027-0.073 Hz) changes in Wilson's disease

Figures 2 and 3 showed the main effects from the two-way repeated-measure ANOVA. Brain regions showing a significant main effect for frequency band were identified in the medial frontal gyrus (Fig. 2, slow-5 > slow-4), inferior temporal gyrus, insula, basal ganglia, hippocampus/ parahippocampal gyrus, and thalamus bilaterally (Fig. 2, slow-5 < slow-4). Figure 3 shows brain regions with a main effect of group, including the cerebellum posterior lobe, inferior temporal gyrus, brain stem, basal ganglia (patient $>$ control), cerebellum anterior lobe and medial frontal gyrus (patient $<$ control). We observed significant interaction between frequency band and group in the cerebellum and left middle frontal gyrus (Fig. 4). Further post-hoc $t$-test revealed that ALFF abnormalities were greater in the slow- 5 than in the slow- 4 band.

\section{Correlation analyses}

The associations between LFO amplitudes and biochemical parameters, i.e., $\mathrm{Cu}$ and $\mathrm{CP}$ were analyzed. $\mathrm{The} \mathrm{Cu}$ level was found to be positively correlated with Slow-5 in the left cerebellum (Fig. 5a), but not with Slow-4. Positive correlations were also found between $\mathrm{CP}$ and ALFF values in the right cerebellum in Slow-5 (Fig. 5b) and in the bilateral cerebellums in Slow-4 (Fig. 5c).

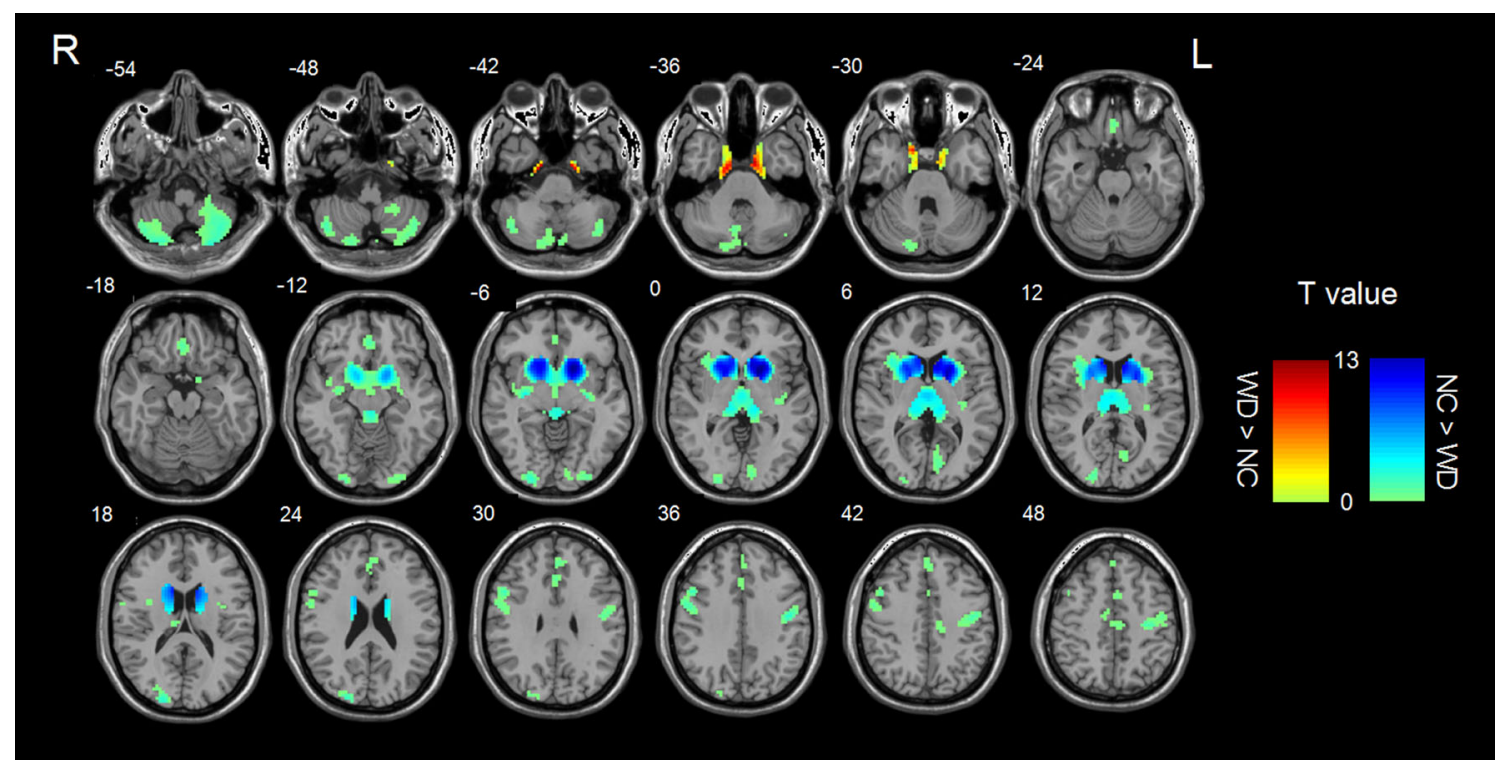

Fig. 1 GM changes in WD patients as compared to normal controls (two-sample t-tests) 
Table 2 Brain regions showing significant GM differences between patients and controls

\begin{tabular}{|c|c|c|c|c|c|}
\hline \multirow[t]{2}{*}{ Brain regions } & \multirow[t]{2}{*}{ Voxels } & \multicolumn{3}{|c|}{ MNI coordinates (mm) } & \multirow[t]{2}{*}{$\mathrm{T}$ value } \\
\hline & & $\mathrm{x}$ & $\mathrm{y}$ & $\mathrm{z}$ & \\
\hline \multicolumn{6}{|l|}{$\mathrm{WD}<\mathrm{NC}$} \\
\hline Basal ganglia & 2281 & -18 & 12 & 0 & -12.39 \\
\hline Left Cerebellum & 291 & -27 & -81 & -57 & -5.06 \\
\hline Right Cerebellum & 277 & 27 & -81 & -54 & -4.07 \\
\hline Left precentral gyrus & 187 & -45 & -12 & 36 & -4.38 \\
\hline Left paracentral lobule & 135 & 0 & -21 & 54 & -4.06 \\
\hline Right middle frontal gyrus & 184 & 51 & 12 & 33 & -4.21 \\
\hline Right medial frontal gyrus & 98 & 3 & 36 & -15 & -3.78 \\
\hline Left medial frontal gyrus & 127 & -3 & 42 & 27 & -3.49 \\
\hline Right superior occipital gyrus & 176 & 21 & -93 & 15 & -4.53 \\
\hline Left middle occipital gyrus & 155 & -30 & -93 & -6 & -3.46 \\
\hline \multicolumn{6}{|l|}{$\mathrm{WD}>\mathrm{NC}$} \\
\hline Left parahippocampal gyrus & 107 & -12 & -6 & -39 & 5.27 \\
\hline Right parahippocampal gyrus & 112 & 15 & -9 & -39 & 5.56 \\
\hline
\end{tabular}

Note: $\mathrm{x}, \mathrm{y}, \mathrm{z}$, coordinates of primary peak locations in the MNI space; $\mathrm{T}$, statistical value of peak voxel showing ALFF differences between two groups. $P<0.05$, corrected for multiple comparisons

\section{Discussion}

In the present study, we examined changes in ALFF in WD patients at different frequency bands (slow-5: $0.01-0.027 \mathrm{~Hz}$; slow-4: $0.027-0.073 \mathrm{~Hz}$ ) with GM correction. We found a general brain atrophy in WD patients, and widespread differences in ALFF of the two bands in many brain regions. Specifically, compared to normal controls, WD patients had increased ALFF in the posterior lobe of the cerebellum, inferior temporal gyrus, brain stem, basal ganglia, and decreased ALFF in the anterior lobe of the cerebellum and medial frontal gyrus, with greater group difference in the slow-5 band than in the slow-4 band in the cerebellum and left middle frontal gyrus. Correlation analysis revealed consistently positive correlations between $\mathrm{Cu}, \mathrm{CP}$ and ALFFs in the cerebellum.

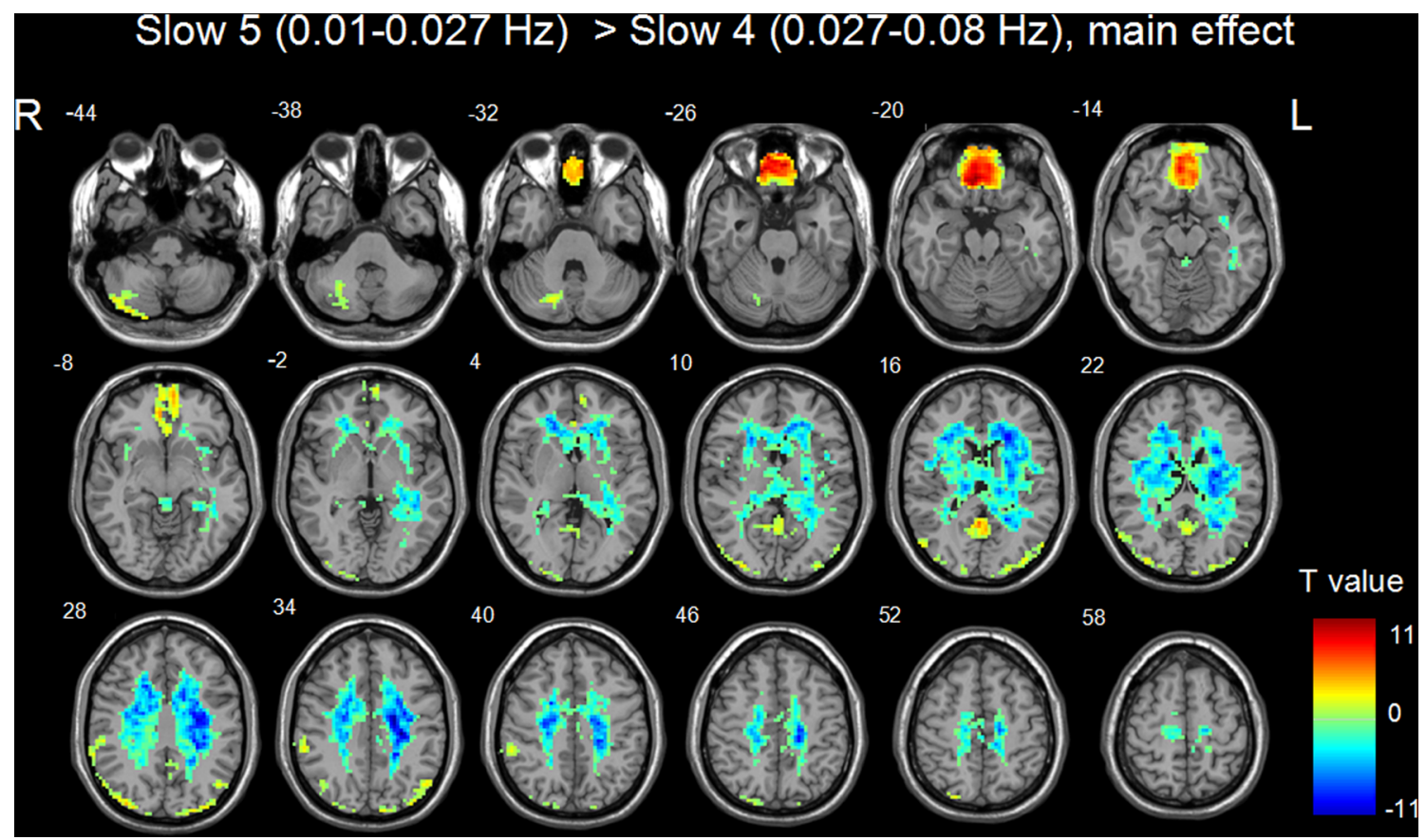

Fig. 2 Frequency-specific ALFF deficits. Hot color represents greater ALFF in the slow-5 band than in the slow-4 band, whereas blue color represents lower ALFF 


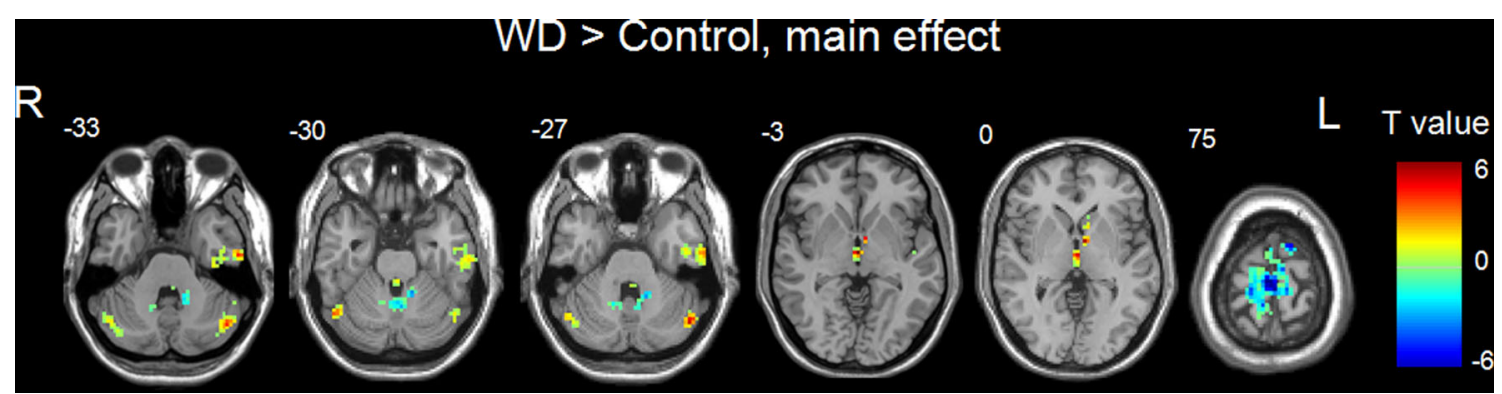

Fig. 3 Regions with ALFF difference between WD and normal groups. Hot color represents higher ALFF in the WD group than in the control group, whereas blue color represents lower ALFF

\section{Changes in GM volume}

The VBM analysis showed that the decrease of GM volumes was most significant in the basal ganglia and were also found in cerebellum, middle and medial frontal gyrus, superior and middle occipital gyrus, precentral and paracentral lobule. The results were consistent with previous MRI studies investigating structural changes in WD patients (Magalhaes et al. 1994; Starosta-Rubinstein et al. 1987), which showed that most obvious lesions were seen in the basal ganglia, and generalized brain atrophy was also common. Because the GM atrophy may lead to artificial reduction in regional ALFF results, all the functional results in the current study have been adjusted with GM correction.

\section{Abnormal ALFF activity in WD patients}

Compared to normal controls, WD patients had increased ALFF in the posterior lobe of the cerebellum, inferior temporal gyrus, brain stem, basal ganglia, and decreased ALFF in the anterior lobe of the cerebellum and medial frontal gyrus. These results suggest specific spontaneous neural activity in different brain regions in WD may have different characteristics from healthy people's. These regions showing abnormal LFO amplitude echoed with the neurological symptoms of WD disease. Previous observations have documented neurological symptoms in WD patients

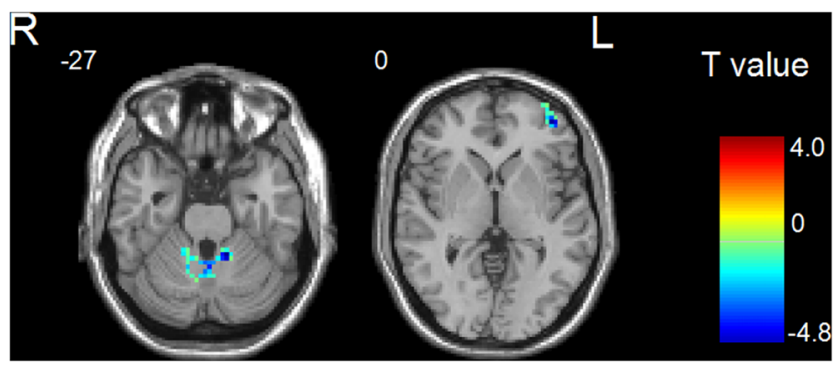

Fig. 4 Areas showing greater difference in slow-5 than in slow-4 between WD and normal groups in the form of parkinsonism (cogwheel rigidity, bradykinesia or slowed movements and a lack of balance are the most common parkinsonian features), masked facial expressions, ataxia (lack of coordination) or dystonia (twisting and repetitive movements of part of the body) (Lorincz 2010). Since lesions of the basal ganglia and cerebellum will lead to motor deficits in animals and humans (Albin et al. 1989; Chesselet and Delfs 1996; Jueptner and Weiller 1998; Barinaga 1996), the abnormal ALFFs observed in basal ganglia and cerebellum are possibly responsible for the motor control or movement-related impairment in WD patients. Starosta-Rubinstein et al. evaluated 31 WD patients with detailed neurologic and medical examinations and found dystonia and bradykinesia correlated with putamen lesions, and dysarthria correlated with both putamen and caudate lesions (Starosta-Rubinstein et al. 1987). On the other hand, the abnormalities in the brainstem may result in changes in sensation, muscle weakness, swallowing and speech difficulty, and co-ordination problems in WD patients (Ertekin et al. 2000; Carmichael et al. 1965). Moreover, the cognition problems (i.e. impulsivity, impaired judgement, promiscuity, apathy and executive dysfunction, slow thinking, and memory loss etc.) and psychiatric problems (i.e. depression, anxiety and psychosis etc.) due to WD (Ala et al. 2007; Lorincz 2010) might result from the joint abnormal functions of the medial frontal gyrus, inferior temporal gyrus and other subcortical regions such as basal ganglia (Ridderinkhof et al. 2004; Chan et al. 2001; Kempton et al. 2011).

In addition, our results showed greater ALFF values for slow-5 band than slow-4 band in the medial frontal gyrus, and greater ALFF values for slow-4 band than slow-5 band in the inferior temporal gyrus, insula, basal ganglia, hippocampus/parahippocampal gyrus, and thalamus, supporting the results by Zuo et al. (2010) revealing that stronger ALFF at slow-5 tended to be present in the cortical structures, and slow-4 tended to be in the subcortical structures. Specifically, we observed that the ALFF abnormalities in the cerebellum and middle frontal gyrus were greater in the slow5 than in the slow-4 band, indicating band-specific abnormal ALFF in WD patients. 
Fig. 5 Areas showing correlation between $\mathbf{a ~} \mathrm{Cu}(\mu \mathrm{mol} /$ day $)$ and slow-5, b CP (mg/dL) and slow-5, c $\mathrm{CP}(\mathrm{mg} / \mathrm{dL})$ and slow-4

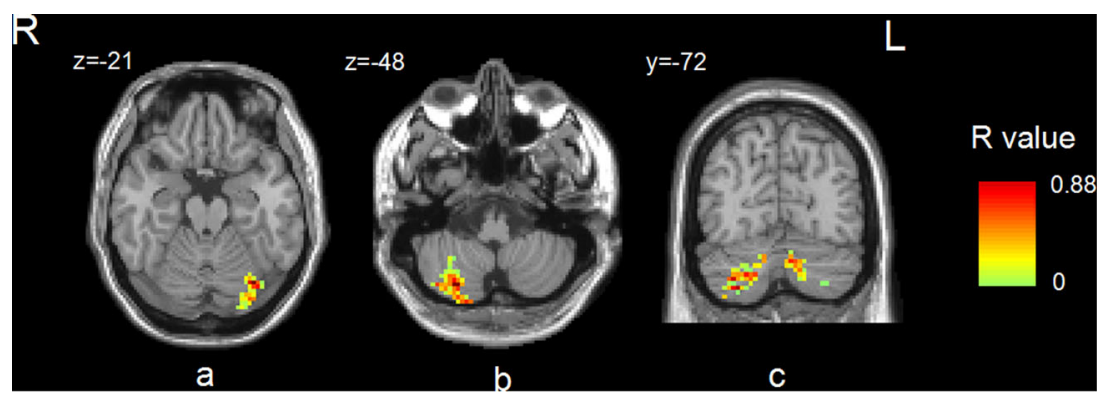

\section{Correlations between LFO amplitudes and biochemical parameters}

To study the associations between LFO amplitudes and biochemical measures could help us better understand the relationship between ALFF values and diagnosis of WD. The results show positive correlations between $\mathrm{Cu}$ and slow-5, $\mathrm{CP}$ and ALFFs at both frequency bands (slow-4 and slow-5) in the cerebellum. It has been shown that most copper is deposited in the basal ganglia of WD, which normally participate in the coordination of movement. Damage to these areas, by Fenton chemistry, produces the neuropsychiatric symptoms seen in Wilson's disease (de Bie et al. 2007). However, we didn't find the correlations between $\mathrm{Cu}, \mathrm{CP}$ and ALFF in the basal ganglia. This may be because all of our ALFF results have been adjusted with GM correction and WD patients have a large GM volume loss in the basal ganglia region as indicated by the VBM analysis. As a result, the information revealed by ALFF is limited in the basal ganglia. By contrast, WD patients showed strong abnormal ALFF activity in large regions of the cerebellum, which area also plays an important role in motor control. Thus, the correlations found between ALFF and biochemical measures such as $\mathrm{Cu}$ and $\mathrm{CP}$ in the cerebellum indicate the WD related changes in the movement function.

Future studies could consider taking patients with hepatic WD as the control, which would be optimal to compare the brain activity changes in the neurological WD. Moreover, it would be interesting to investigate ALFF changes before and after the treatment, and to correlate the ALFF changes with the clinical outcome. All of our WD patients were treated with DMPS, which is widely used in China for more than 30 years (Chen et al. 2016; Yang and Cheng 2002; Wang et al. 2003). We noted that clinical practice with WD patient in Europe and the United States used Penicillamine instead of DMPS (European Association For The Study Of The Liver 2012). Although the general mechanisms underlying DMPS and Penicillamine might be similar (Gerhardsson and Aaseth 2016), whether our findings with ALFF abnormalities were universal characteristics of WD patient required further investigation.

\section{Conclusion}

This study shows that WD patients had abnormal LFO amplitude in many brain regions, including the cerebellum, inferior temporal gyrus, brain stem, basal ganglia, and medial frontal gyrus, with different spatial patterns in different frequency bands, and there was a correlation between LFO amplitudes and biochemical measures in the cerebellum. Thus, the accumulation of copper profoundly impaired intrinsic brain activity and the impairments seem to be frequency-dependent. These results provide further insights into the understanding of the pathophysiology of WD.

Acknowledgements This study was supported by the Research Project of Anhui Province (1301042202) awarded to Yongqiang Yu and the Knowledge Innovation Program of the Chinese Academy of Sciences (Y3CX102003), Institute of Psychology, CAS (Y1CX201006) awarded to Chang-Bing Huang.

Open Access This article is distributed under the terms of the Creative Commons Attribution 4.0 International License (http:// creativecommons.org/licenses/by/4.0/), which permits unrestricted use, distribution, and reproduction in any medium, provided you give appropriate credit to the original author(s) and the source, provide a link to the Creative Commons license, and indicate if changes were made.

\section{References}

Ala A, Walker AP, Ashkan K, Dooley JS, Schilsky ML (2007) Wilson's disease. Lancet 369(9559):397-408

Albin RL, Young AB, Penney JB (1989) The functional anatomy of basal ganglia disorders. Trends Neurosci 12(10):366-375

Ashburner J, Friston KJ (2005) Unified segmentation. NeuroImage 26: 839-851

Barinaga M (1996) The cerebellum: movement coordinator or much more? Science 272(5261):482-483

Biswal B, Yetkin FZ, Haughton VM, Hyde JS (1995) Functional connectivity in the motor cortex of resting human brain using echo-planar MRI. Magn Reson Med 34(4):537-541

Carmichael EA, Dix MR, Hallpike CS (1965) Observations upon the neurological mechanism of directional preponderance of caloric nystagmus resulting from vascular lesions of the brain-stem. Brain 88(1):51-74

Chan D, Fox NC, Crum WR et al (2001) Patterns of temporal lobe atrophy in semantic dementia and Alzheimer's disease. Ann Neurol 49(4):433-442 
Chao-Gan Y, Yu-Feng Z (2010) DPARSF: a MATLAB toolbox for "pipeline" data analysis of resting-state fMRI. Front Syst Neurosci 4:13

Chen D, Zhou X, Hou H et al (2016) Clinical efficacy of combined sodium dimercaptopropanesulfonate and zinc treatment in neurological Wilson's disease with D-penicillamine treatment failure. Ther Adv Neurol Disord 9(4):310-316

Chesselet MF, Delfs JM (1996) Basal ganglia and movement disorders: an update. Trends Neurosci 19(10):417-422

Collignon A, Maes F, Delaere D, Vandermeulen D, Suetens P, Marchal G (1995) Automated multi-modality image registration based on information theory. In: Bizais Y, Barillot C, Di Paola R (eds) Information processing in medical imaging. Kluwer Academic Publishers, Dordrecht (The Netherlands), pp 263-274

de Bie P, Muller P, Wijmenga C, Klomp LW (2007) Molecular pathogenesis of Wilson and Menkes disease: correlation of mutations with molecular defects and disease phenotypes. J Med Genet 44(11):673-688

Ertekin C, Aydogdu I, Yüceyar N, Kiylioglu N, Tarlaci S, Uludag B (2000) Pathophysiological mechanisms of oropharyngeal dysphagia in amyotrophic lateral sclerosis. Brain 123(1):125-140

European Association For The Study Of The Liver (2012) EASL clinical practice guidelines: Wilson's disease. J Hepatol 56(3):671-685

Gerhardsson L, Aaseth J (2016) Chapter 7 - Guidance for Clinical Treatment of Metal Poisonings-Use and Misuse of Chelating Agents. In Chelation Therapy in the Treatment of Metal Intoxication (pp. 313-341). Boston: Academic Press

Hawkins RA, Mazziotta JC, Phelps ME (1987) Wilson's disease studied with FDG and positron emission tomography. Neurology 37(11):1707

He Y, Wang L, Zang Y et al (2007) Regional coherence changes in the early stages of Alzheimer's disease: a combined structural and resting-state functional MRI study. NeuroImage 35:488-500

Hoptman MJ, Zuo XN, Butler PD et al (2010) Amplitude of lowfrequency oscillations in schizophrenia: a resting state fMRI study. Schizophr Res 117:13-20

Jueptner M, Weiller C (1998) A review of differences between basal ganglia and cerebellar control of movements as revealed by functional imaging studies. Brain 121(8):1437-1449

Kempton MJ, Salvador Z, Munafò MR, Geddes JR, Simmons A, Frangou S (2011) Structural neuroimaging studies in major depressive disorder: meta-analysis and comparison with bipolar disorder. Arch Gen Psychiatry 68(7):675-690

Kuwert T, Hefter H, Scholz D et al (1992) Regional cerebral glucose consumption measured by positron emission tomography in patients with Wilson's disease. Eur J Nucl Med 19(2):96-101

Lorincz MT (2010) Neurologic Wilson's disease. Ann N Y Acad Sci 1184:173-187

Machado A, Chien HF, Deguti MM et al (2006) Neurological manifestations in Wilson's disease: report of 119 cases. Mov Disord 21:2192-2196

Magalhaes ACA, Caramelli P, Menezes JR et al (1994) Wilson's disease: MRI with clinical correlation. Neuroradiology 36(2):97-100

Packard MG, Knowlton BJ (2002) Learning and memory functions of the basal ganglia. Annu Rev Neurosci 25(1):563-593

Page RA, Davie CA, MacManus D et al (2004) Clinical correlation of brain MRI and MRS abnormalities in patients with Wilson disease. Neurology 63:638-643
Piga M, Murru A, Satta L et al (2008) Brain MRI and SPECT in the diagnosis of early neurological involvement in Wilson's disease. Eur J Nucl Med Mol Imaging 35(4):716-724

Radua J, van den Heuvel OA, Surguladze S, Mataix-Cols D (2010) Metaanalytical comparison of voxel-based morphometry studies in obsessive-compulsive disorder vs other anxiety disorders. Arch Gen Psychiatry 67(7):701-711

Reilly M, Daly L, Hutchinson M (1993) An epidemiological study of Wilson's disease in Ireland. J Neurol Neurosurg Psychiatry 56: 298-300

Ridderinkhof KR, Ullsperger M, Crone EA, Nieuwenhuis S (2004) The role of the medial frontal cortex in cognitive control. Science 306(5695):443-447

Sinha S, Taly AB, Ravishankar S et al (2006) Wilson's disease: cranial MRI observations and clinical correlation. Neuroradiology 48(9): 613-621

Song XW, Dong ZY, Long XY, Li SF, Zuo XN, Zhu CZ, He Y, Yan CG, Zang YF (2011) REST: a toolkit for resting-state functional magnetic resonance imaging data processing. PLoS One 6(9):e25031

Starosta-Rubinstein S, Young AB, Kluin K et al (1987) Clinical assessment of 31 patients with Wilson's disease: correlations with structural changes on magnetic resonance imaging. Arch Neurol 44(4): 365-370

Turner RS, Desmurget M, Grethe J, Crutcher MD, Grafton ST (2003) Motor subcircuits mediating the control of movement extent and speed. J Neurophysiol 90(6):3958-3966

Wang XP, Yang RM, Ren MS, Sun BM (2003) Anticopper efficacy of captopril and sodium dimercaptosulphonate in patients with Wilson's disease. Funct Neurol 18(3):149-154

Wang Z, Zhang Z, Liao W et al (2014) Frequency-dependent amplitude alterations of resting-state spontaneous fluctuations in idiopathic generalized epilepsy. Epilepsy Res 108(5):853-860

Wilson SA (1912) Dégénérescence lenticulaire progressive, maladie nerveuse familiale associée à la cirrhose du foie. Rev Neurol 23: 229-233

Yamada H, Matsumoto N, Kimura M (2004) Tonically active neurons in the primate caudate nucleus and putamen differentially encode instructed motivational outcomes of action. J Neurosci 24(14): 3500-3510

Yang RM, Cheng N (2002) Observation on short-term effect and follow-up of integrative medicine in treating 198 patients with hepatolenticular degeneration. CJIM 8:294

Yin Y, Li L, Jin C et al (2011) Abnormal baseline brain activity in posttraumatic stress disorder: a resting-state functional magnetic resonance imaging study. Neurosci Lett 498(3):185-189

Zang YF, He Y, Zhu CZ et al (2007) Altered baseline brain activity in children with ADHD revealed by resting-state functional MRI. Brain Dev 29:83-91

Zou K, Deng W, Li T et al (2010) Changes of brain morphometry in firstepisode, drug-naive, non-late-life adult patients with major depression: an optimized voxel-based morphometry study. Biol Psychiatry 67:186-188 